\title{
Divulgation et rendement : on s'attend à plus et mieux
}

$\mathrm{C}$ eux et celles d'entre nous qui démarrent leur journée par la double attaque contre le système nerveux que constituent la tasse de café et le journal du matin commencent à s'habituer aux mauvaises nouvelles dans le domaine des soins de santé. En voici un exemple : le journaliste Barry Meier a révélé récemment dans le New York Times que l'on savait, plus de trois ans avant que les organismes de réglementation fédéraux diffusent des avertissements à cet égard, qu'un défibrillateur interne à synchronisation automatique pouvait mal fonctionner ${ }^{1}$. Les dirigeants de l'entreprise ont déclaré n'avoir pas informé les patients d'un défaut électrique dans un faible pourcentage d'unités sous prétexte que la publicité risquait de faire plus de mal que de bien étant donné le faible pourcentage d'appareils défectueux et les risques posés par l'intervention chirurgicale nécessaire pour les remplacer ${ }^{2}$. Ainsi, Joshua Oukrop, âgé de 21 ans, mort pendant une randonnée à bicyclette à la suite d'une panne de son défibrillateur implanté, n'a jamais eu la possibilité de peser les risques lui-même.

Même s'il est déplorable et digne de mention dans les médias qu'une entreprise (sans oublier la FDA et Santé Canada) n'a pas divulgué en temps opportun de l'information sur le rendement de l'appareil (en espérant que l'on prendra des mesures correctives), on suivait au moins le rendement dans ce cas. Le rendement de la prestation de soins médicaux de routine, à l'hôpital ou au cabinet du médecin, est laissé quant à lui presque entièrement sans surveillance. Et l'on ne peut divulguer ce qu'on ne surveille pas.

Les hôpitaux où l'on trouve des patients gravement malades qui ont besoin de soins médicaux complexes sont des endroits particulièrement dangereux. Le taux global d'incidence d'événements indésirables causant la mort, une incapacité ou une hospitalisation prolongée dans les hôpitaux du Canada s'établit à 7,5 pour 100 hospitalisations, dont $37 \%$ sont évitables (intervalle de confiance à $95 \%, 32 \%-42 \%$ ), ce qui représente un total estimatif de 70000 événements indésirables évitables graves par année 3 .

Si ces statistiques déconcertent le public et les médecins, nous ferions bien de tenir compte de la façon insidieuse dont peuvent surgir l'erreur médicale et les événements indésirables. L'Étude sur les événements indésirables au Canada a signalé dans une grande mesure les erreurs de diagnostic, la prescription de médicaments contre-indiqués et la prise en charge incorrecte de défaillances d'organes.

Il y a toutefois d'autres défaillances, peut-être plus courantes, que l'on ne surveille pas. Il s'agit du défaut de prendre en charge les patients conformément à des normes de soins généralement reconnues. Ces omissions ne causeront peut-être pas d'événements indésirables au cours d'une hospitalisation, mais elles exposent tout de même inutilement les patients à un risque accru. Nous savons, par exemple, qu'il faut prescrire à des patients qui ont subi un infarctus aigu du myocarde des $\beta$-bloquants et de l'AAS, à moins de contre- indications précises. Or, certains patients reçoivent des soins qui reflètent fidèlement les recommandations des guides, mais d'autres pas : cette application des normes factuelles est problématique. Ce type de problème passif de qualité des soins n'attire pas beaucoup l'attention des médias. Les patients ne savent pas non plus nécessairement s'ils reçoivent des soins de qualité inférieure.

Bien entendu, pour une grande partie de la pratique de la médecine, qui repose sur la capacité de chaque médecin de personnaliser les décisions de traitement en fonction des besoins de chaque patient, il n'existe aucune formule précise. Cependant, pour beaucoup de problèmes, les normes généralement reconnues sont applicables à presque tous les groupes de patients. On pourrait mettre en œuvre ces normes sur le processus de soin dans les services hospitaliers et ambulatoires, en surveiller l'observation et en divulguer les résultats. Les patients et le public peuvent comprendre les nuances, les rajustements du risque en fonction de la gravité de la maladie, et utiliser judicieusement ces renseignements. Il y a encore plus important : la divulgation du processus et des résultats poussera les médecins et d'autres prestateurs à améliorer leur rendement en prodiguant des soins qui reflètent plus clairement les normes généralement reconnues.

Aux États-Unis, on tente de surveiller la pratique dans le cas des patients qui ont des problèmes particuliers et d'accréditer les médecins qui ont réussi à atteindre des normes établies dans la pratique réelle. Ces résultats sont divulgués (voir www.ncqa .org/PhysicianQualityReports.htm). Dans le cas des hôpitaux, la surveillance est encore plus facile que dans celui des soins ambulatoires. Il existe déjà, dans les résumés de congé des patients, des dossiers consultables électroniquement sur les soins et les résultats. Ces dossiers permettent de comparer le rendement des hôpitaux pour plusieurs problèmes importants comme la prise en charge de l'infarctus aigu du myocarde, de la pneumonie, de l'insuffisance cardiaque globale et de la grossesse. Aux ÉtatsUnis, donc, on a établi des normes, on suit le rendement et on divulgue les résultats (voir www.jcaho.org et www.cms.hhs .gov/quality/hospital). La divulgation publique a obligé les hôpitaux non performants à s'améliorer".

Au Canada, ces données, si elles existent, ne sont pas divulguées. Ne nous laissons pas distraire par les manchettes. Il y a beaucoup de travail à faire dans la pratique quotidienne de la médecine. - $7 A M C$

\section{Références}

1. Meier B. Maker of heart device kept flaw from doctors. New York Times 2005 May 24;Sect A:1.

2. Steinbrook R. The controversy over Guidant's implantable defibrillators. $N$ Engl 7 Med 2005;353(3):221-4.

3. Baker GR, Norton PG, Flintoft V, Blais R, Brown A, Cox J, et al. The Canadian Adverse Events Study: the incidence of adverse events among hospital patients in Canada. FAMC 2004;170(11):1678-86.

4. Jha AK, Li Z, Orav EJ, Epstein AM. Care in US hospitals - the hospital quality alliance program. $N$ Engl 7 Med 2005;353(3):265-74. 